\title{
De la cessation d'activité
}

\section{Eberhard Wolff}

Prof. Dr rer. soc., rédacteur culture, histoire, société

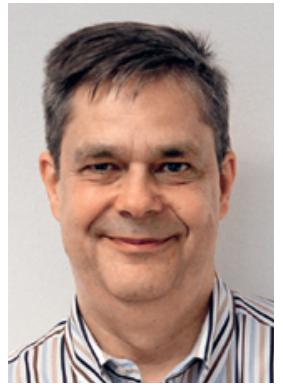

Interview de Pau Simon pour l'émission de radio NPR "All Songs Considered» le 5 septembre 2018. A écouter en ligne.

2 Seidenberg A. Heute ist der Tag: 5.4.2018. Bull Med Suisses. 2018;99(16):509.

Tanner M, Christen P. «Personne ne veut re prendre mon cabinet». Bull Med Suisses. 2017;98(47):1572-3.

4 Oertle D. Zukunft nach der Praxis. Bull Med Suisses. 2018;99(15): 491-2.

Voir l'exemple du pédiatre zurichois Willy Krauthammer, 88 ans, dans Hudec J. Paradox in der Pädiatrie (...). Neue Zürcher Zeitung, 9.10.2018.

6 Grete W. Ein Rezepturverbot für Ärztinnen und Ärzte im Ruhestand? Bull Med Suisses. 2018;99(38): 1295.

Thorwald J. La fin d'un grand chirurgien. Paris: Albin Michel; 1962

eberhard.wolff[at]saez.ch
Brusquement, un interrupteur a fait «clic» dans sa tête et il a su: "Maintenant, j'en ai fini avec mon travail. Il est temps d'arrêter, après plus de soixante ans de pratique.» Non, je ne parle ni d'un médecin de premier recours ni d'un cardiochirurgien émérite, mais de Paul Simon, 77 ans, et de son métier d'écrire des chansons. C'est ainsi qu'il a récemment expliqué sa décision lors d'une interview radiophonique [1]. La tournée d'adieu est désormais terminée. Le site Web dédié à ses tournées est vide.

Ce qui semble si simple et convaincant dans son cas est un cap extrêmement complexe, souvent douloureux, y compris, voire surtout dans le domaine médical. Cela se reflète dans de nombreux articles récents du BMS. Quand et comment voulons-nous cesser d'exercer notre profession, quand avons-nous la possibilité de le faire, le droit de le faire, le devoir de le faire? Nous pouvons en fait décliner la problématique de l'arrêt sur tout l'éventail des marqueurs de modalité qui complètent le verbe principal par des notions de possibilité et de nécessité.

L'un des avantages bien connus des médecins libéraux est qu'ils ne sont pas obligés d'arrêter à 65 ans s'ils ne le veulent pas. Mais s'ils veulent malgré tout s'arrêter [2] parce que leur cabinet est constamment bondé, il se peut tout à fait qu'ils ne le puissent pas ou, dans l'intérêt des soins aux patients, qu'ils ne soient pas autorisés à s'arrêter faute de successeur approprié pour reprendre le cabinet [3].

Il en va tout autrement des médecins du secteur public. Tous ceux qui veulent arrêter en ont le droit et le peuvent. A tout moment, dès lors qu'ils peuvent se le permettre. A contrario, s'ils ne veulent pas arrêter, ils ont souvent un problème, parce que notre système de travail est toujours régi par l'idée qu'ils devraient et de facto souvent même qu'ils ont l'obligation d'arrêter à l'«âge normal de départ à la retraite». Malgré quelques exceptions récentes, la notion archaïque qu'après précisément 65 (ou 64) révolutions complètes de la terre autour du soleil depuis la naissance et après des décennies de travail actif, tout doit cesser du jour au lendemain, reste encore très vivace. On doit passer instantanément de cent à zéro, du moins en principe. Mais, bien avant leur retraite déjà, ces médecins sont de plus en plus nombreux à se dire qu'ils ne veulent pas arrêter si brutalement, mais continuer de travailler sous une forme ou une autre, parce que la perspective d'une vie sans activité professionnelle les inquiète toujours davantage [4]. Ils deviennent alors médecins libéraux, pour certains jusqu'à un âge avancé [5]. J'aimerais moimême continuer à travailler en tant que rédacteur, professeur d'université et scientifique établi sur des projets de recherche dans six ans.

Au début de l'année, le canton de Zurich a supprimé les «autorisations senior» qui permettaient aux médecins retraités de soigner leur famille et leurs amis proches et de leur établir des ordonnances [6]. Les médecins libéraux ont ainsi aussi eu un aperçu de ce que cela signifie d'être obligés d'arrêter complètement une fois à la retraite. La résistance s'organise.

Et il y a ceux qui ne peuvent pas arrêter. Pour des raisons économiques, par exemple. Aux Etats-Unis, des professeurs d'université continuent pour certains de travailler (sans limite d'âge) pour ne pas perdre leur assurance-maladie. D'autres ne veulent pas renoncer aux avantages de leur profession, comme la reconnaissance. Le chirurgien berlinois Ferdinand Sauerbruch (1875-1951, à Zurich de 1910 à 1918) a apparemment continué à opérer malgré une sclérose cérébrale avancée, multipliant les erreurs. Un ouvrage à scandale racoleur a rendu l'affaire publique en 1960 [7].

Arrêter n'est donc pas toujours aussi simple que Paul Simon l'a décrit avec aisance et légèreté. Ozzy Osbourne, le prince des ténèbres septuagénaire du heavy metal, parcourt actuellement le monde à l'occasion de sa tournée d'adieu. Avec un brin d'humour, elle a été baptisée "No More Tours 2», puisqu'il avait déjà fait une première tournée d'adieu en 1992. Quant à savoir s'il s'agit cette fois vraiment de la dernière, il laisse délibérément la question en suspens. On ne sait pas très bien s'il cultive ce flou parce qu'il n'est pas obligé d'arrêter, ne le veut pas, n'en a pas le droit ou ne le peut pas. Et Paul Simon? Je suis convaincu qu'il continuera à faire de la musique, donner des interviews et s'investir dans des projets aussi longtemps qu'il le pourra. Et c'est exactement ce que veulent de nombreux médecins et autres actifs.

On devrait aujourd'hui laisser celles et ceux qui ne veulent pas vraiment arrêter faire ce qu'ils ou elles sont encore capables de faire. En 2016, dans un état multimorbide, Leonhard Cohen n'a pu enregistrer son tout à fait remarquable titre d'adieu "You Want It Darker» qu'à son domicile. Il est sorti 19 jours avant sa mort. 